\title{
HOMOGENEOUS SOLUTION OF A NONLINEAR DIFFERENTIAL EQUATION
}

\author{
JAMES L. REID
}

AbStRaCt. The nonlinear second order differential equation satisfied by the homogeneous function $y=\left[a u^{m}+m b u^{j} v^{n}+c v^{m}\right]^{k / m}$, $m=j+n$, is obtained. Functions $u$ and $v$ satisfy independently the linear equation $\ddot{y}+r(t) \dot{y}+q(t) y=0$. The nonlinear equation derived contains previous results as special cases of $r(t)$, of the constants $a$, $b$, and $c$, and of the numbers $k$ and $m$.

Two linearly independent solutions $u(t)$ and $v(t)$ are assumed to satisfy the linear homogeneous differential equation

$$
\ddot{y}+r(t) \dot{y}+q(t) y=0,
$$

where dots indicate differentiation with respect to the independent variable $t$. A limited number of nonlinear differential equations have exact solutions which are constructed in terms of $u$ and $v$. The equation derived by Pinney [1] can easily be extended to include the first derivative term, i.e.,

$$
\ddot{y}+r(t) \dot{y}+q(t) y=a c W^{2} y^{-3},
$$

with the solution

$$
y=\left[a u^{2}+c v^{2}\right]^{1 / 2},
$$

where the Wronskian $W=u \dot{v}-v \dot{u}$ is a function of $t$ and the constants $a$ and $c$ are arbitrary. Likewise the results of [2] are readily modified to include the case $r(t) \neq 0$ and $a \neq 1$, the generalization being

$$
\ddot{y}+r(t) \dot{y}+q(t) y=a c(m-1)(u v)^{m-2} W^{2} y^{1-2 m},
$$

where the solution is

$$
y=\left[a u^{m}+c v^{m}\right]^{1 / m} .
$$

The number $m$ is assumed to be real and nonzero. Thomas [3] has shown that the function

$$
y=(u v)^{k / 2}
$$

Received by the editors November 12, 1971 and, in revised form, March 24, 1972. AMS (MOS) subject classifications (1970). Primary 34A05, 46L05.

Key words and phrases. Exact homogeneous solution, nonlinear second order differential equation, homogeneous linear differential equation.

(C) American Mathematical Society 1973 
satisfies the nonlinear equation

$$
\ddot{y}+r(t) \dot{y}+k q(t) y=(1-l) \dot{y}^{2} y^{-1}-\frac{1}{4} k W^{2} y^{1-4 l}
$$

where $k$ is assumed real and nonzero and $k l=1$. Equation (7) is the form listed by Herbst [4], and it will be referred to as Thomas' equation. Direct substitution easily verifies that (6) satisfies (7).

Solutions (1) and (3) are homogeneous of degree one in $u$ and $v$, while (6) is homogeneous of degree $k$. The object of this note is to derive a nonlinear equation, having a solution homogeneous of degree $k$ in $u$ and $v$, that contains the results above as special cases.

Before progressing to the general homogeneous function (19), it is useful to consider the special case

$$
y=\left[a u^{m}+c v^{m}\right]^{k / m},
$$

since it is easier to follow in detail. The steps in the derivation of the general case will be seen to be analogous to this example. The function given by (8) is to satisfy a nonlinear differential equation of the form

$$
\ddot{y}+r(t) \dot{y}+k q(t) y=R(t),
$$

where $R$ represents the nonlinear terms to be determined. The first and second derivatives of $y$ are written as

$$
\begin{gathered}
\dot{y}=k y^{1-m l} A, \\
\ddot{y}=k(k-m) y^{1-2 m l} A^{2}+k y^{1-m l} \dot{A},
\end{gathered}
$$

respectively, where $k l=1$ and $A$ is

$$
A=a u^{m-1} \dot{u}+c v^{m-1} \dot{v} .
$$

In terms of $A$, (9) can be expressed as

$$
k(k-m) y^{1-2 m l} A^{2}+k y^{1-m l}\left[\dot{A}+r(t) A+q(t) y^{m l}\right]=R .
$$

Multiplying (13) by $y^{2 m l-1}$ and substituting for $A, \dot{A}$ and $y^{m l}$ in the bracket gives

$$
\begin{aligned}
k(k-m) A^{2}+k y^{m l}[ & (m-1)\left(a u^{m-2} \dot{u}^{2}+c v^{m-2} \dot{v}^{2}\right) \\
& +(\ddot{u}+r(t) \dot{u}+q(t) u) a u^{m-1} \\
& \left.+(\ddot{v}+r(t) \dot{v}+q(t) v) b v^{m-1}\right]=y^{2 m l-1} R .
\end{aligned}
$$

By assumption $u$ and $v$ satifsy (1) and, hence, the coefficients of $a u^{m-1}$ and $b v^{m-1}$ are zero. It is now convenient to write (14) as

$$
k^{2} A^{2}-m k A^{2}+k(m-1) y^{m l}\left(a u^{m-2} \dot{u}^{2}+c v^{m-2} \dot{v}^{2}\right)=y^{2 m l-1} R
$$


The last two terms on the left side of (15) can be combined by using $W=$ $u \dot{v}-v \dot{u}$ and (12) again for $A$ to obtain

$$
k(k-1) A^{2}+k(m-1) a c(u v)^{m-2} W^{2}=y^{2 m l-1} R .
$$

Now, $A=l \dot{y} y^{m l-1}$ from (10) can be used in (16) to yield an identity for $R$, i.e., if

$$
(1-l) \dot{y}^{2} y^{2 m l-2}+k(m-1) a c(u v)^{m-2} W^{2}=y^{2 m l-1} R,
$$

then by inspection

$$
R=(1-l) \dot{y}^{2} y^{-1}+k(m-1) a c(u v)^{m-2} W^{2} y^{1-2 m l} .
$$

This completes the derivation for the function given by (8). Clearly, the nonlinear equations resulting from (3) and (5) can be verified in this manner.

If a third term analogous to (6) is considered, the homogeneous function can be written as

$$
y=\left[a u^{m}+m b u^{j} v^{n}+c v^{m}\right]^{k / m}, \quad m=j+n,
$$

where $b$ is an arbitrary constant. For this case, the derivatives in (9) may be expressed as

$$
\begin{gathered}
\dot{y}=k y^{1-m l} C, \\
\ddot{y}=k(k-m) y^{1-2 m l} C^{2}+k y^{1-m l} \dot{C},
\end{gathered}
$$

where $k l=1$ and $C$ is defined by

$$
C=A+b u^{j-1} v^{n-1} B,
$$

with $A$ given by (12) and $B$ defined by

$$
B=j \dot{u} v+n \dot{u} v .
$$

The equation analogous to (13) is

$$
k(k-m) y^{1-2 m l} C^{2}+k y^{1-m l}\left[\dot{C}+r(t) C+q(t) y^{m l}\right]=F,
$$

where $F$ is to be determined. The remainder of the calculation is the straightforward, but rather lengthy, evaluation of (13a). The result corresponding to (15) is

$$
\begin{aligned}
& k(k-m) C^{2}+ k(m-1) y^{m l} \\
&(15 \mathrm{a})+\left\{a u^{m-2} \dot{u}^{2}+c v^{m-2} \dot{v}^{2}+b m u^{j-1} v^{n-1} \dot{u} \dot{v}\right. \\
&\left.\cdot b\left[(j-1) u^{j-2} v^{n-2} \dot{u}+(n-1) u^{j-1} v^{n-2} \dot{v}\right] B\right\}=y^{2 m l-1} F,
\end{aligned}
$$


and that corresponding to (18) is

$$
\begin{aligned}
F= & (1-l) \dot{y}^{2} y^{-1} \\
(18 \mathrm{a})+ & \left\{k(m-1) a c(u v)^{m-2} W^{2}-k b^{2} n j u^{2 j-2} v^{2 n-2} W^{2}\right. \\
& \left.+k b\left[(m-j-1) n a u^{m}+(m-n-1) n c v^{m}\right] u^{j-2} v^{n-2} W^{2}\right\} y^{1-2 m l} .
\end{aligned}
$$

The main result of this note can now be stated: The homogeneous function given by (19) satisfies the nonlinear differential equation

$$
\ddot{y}+r(t) \dot{y}+k q(t) y=(1-l) \dot{y}^{2} y^{-1}+k Q W^{2} y^{1-2 m l},
$$

where $k l=1$ and

$$
\begin{aligned}
Q= & b u^{j-2} v^{n-2}\left[(m-j-1) n a u^{m}+(m-n-1) j c v^{m}-b n j u^{j} v^{n}\right] \\
& +(m-1) a c(u v)^{m-2} .
\end{aligned}
$$

When the numbers $a=c=0, m=2, j=n=1$, and $b=\frac{1}{2}$ are substituted into (19), (24) and (25), Thomas' results expressed by (6) and (7) are obtained. The Pinney equation (2) arises when $b=0, k=1$, and $m=2$, while (4) and (5) are regained with $b=0$ and $k=1$. The result (18) clearly follows for $b=0$ in (18a).

Some additional special cases are pointed out. For $m=2$, and $b=0$ in (24) and (25), one gets a generalization of the Pinney equation similar to Thomas', i.e.,

$$
\ddot{y}+r(t) \dot{y}+k q(t) y=(1-l) \dot{y}^{2} y^{-1}+k a c W^{2} y^{1-4 l}
$$

a solution of which follows from (19).

The final generalization of the Pinney equation allowed by (24) and (25) results when $m=2$ and the requirement that $n+j=2$. The case for $j=n=$ $m / 2$ follows from (25) by replacing $m b$ by $b, Q$ becoming

$$
Q^{\prime}=(b / 4)\left[(m-2)\left(a u^{m}+c v^{m}\right)(u v)^{-m / 2}-b+4 b^{-1}(m-1) a c\right](u v)^{m-2} .
$$

The solution of the nonlinear equation

$$
\ddot{y}+r(t) \dot{y}+k q(t) y=(1-l) \dot{y}^{2} y^{-1}+k Q^{\prime} W^{2} y^{1-2 m l},
$$

where $k l=1$, is therefore

$$
y=\left[a u^{m}+b(u v)^{m / 2}+c v^{m}\right]^{k / m} .
$$

It is interesting to note that the choice $m=2, k=1$, and $r(t)=0$ in (27), (28) and (29) is equivalent to the equation and solution obtained by Lewis [5]. For this case $Q^{\prime}$ reduces to the constant $\left(a c-b^{2} / 4\right)$ and the Wronskian also is constant. Indeed, when the coefficient $r(t)$ is identically zero the Wronskian is a constant in all the equations above, and it can be incorporated into the solutions as was done in [1], [2], and [5]. 
The parameters $k$ and $m$ allow (24) to encompass a variety of physical problems, while the arbitrary constants $a, b$, and $c$ can be chosen to fit a particular case. Lewis [5] has developed an invariant of the classical and the quantum oscillator based on (29) above. Kostin [6] has derived nonlinear equations for the probability density of quantum theory; solutions to his equations can be found as special cases of (9) with $R$ given by (18). To illustrate the use of (24) and (25), particular solutions of the Duffing-type equations

$$
\ddot{y} \pm \omega^{2} y \mp \beta^{2} e^{-6 \omega t} y^{3}=0
$$

are noted, where $\omega$ and $\beta$ are constants. For $+\omega^{2}$, the solution

$$
y=\sqrt{ } 10(\omega / \beta) e^{3 \omega t}
$$

follows for $m=-1, j=(3 i-1) / 2, n=-(3 i+1) / 2$, where $i=\sqrt{ }-1$, and for $a=c=0, b^{2}=\beta^{2} / 10 \omega^{2}$, and $W^{2}=-4 \omega^{2}$. For $-\omega^{2}$, the solution

$$
y=e^{3 \omega t} /\left(a e^{2 \omega t}-\beta / 2 \omega\right)
$$

results for $m=-1, j=-2, n=1$ and for $c=0, b^{2}=\beta^{2} / 4 \omega^{2}$, and $W^{2}=$ $4 \omega^{2}$. The constant $a$ can be determined from an initial condition, e.g., $a=1 / y_{0}+\beta / 2 \omega$, where $y_{0}$ is some nonzero initial amplitude.

\section{REFERENCES}

1. E. Pinney, The nonlinear differential equation $y^{\prime \prime}+p(x) y+c y^{-3}=0$, Proc. Amer. Math. Soc. 1 (1950), 681. MR 12, 336.

2. J. L. Reid, An exact solution of the nonlinear differential equation $\ddot{y}+p(t) y=$ $q(t) / y^{2 m-1}$, Proc. Amer. Math. Soc. 27 (1971), 61-62. MR 42 \#4800.

3. J. M. Thomas, Equations equivalent to a linear differential equation, Proc. Amer. Math. Soc. 3 (1952), 899-903. MR 14, 558.

4. R. T. Herbst, The equivalence of linear and nonlinear differential equations, Proc. Amer. Math. Soc. 7 (1956), 95-97. MR 17, 848.

5. H. R. Lewis, Jr., Classical and quantum systems with time-dependent harmonicoscillator-type Hamiltonians, Phys. Rev. Lett. 18 (1967), 510.

6. M. D. Kostin, Linear differential equation for the radial distribution function of quantum mechanics, J. Chem. Phys. 54 (1971), 2739.

Physics Department, Clemson University, Clemson, South Carolina 29631 